\title{
HDAC4 Regulates Skeletal Muscle Regeneration via Soluble Factors
}

\author{
Alessandra Renzini ${ }^{1+}$, Nicoletta Marroncelli ${ }^{1 \dagger}$, Chiara Noviello ${ }^{1}$, Viviana Moresi ${ }^{1,2 *}$ and \\ Sergio Adamo' \\ ${ }^{1}$ DAHFMO Unit of Histology and Medical Embryology, Interuniversity Institute of Myology, Sapienza University of Rome, \\ Rome, Italy, ${ }^{2}$ Laboratory of Cardiovascular Endocrinology, IRCCS San Raffaele Pisana, Rome, Italy
}

Skeletal muscle possesses a high ability to regenerate after an insult or in pathological conditions, relying on satellite cells, the skeletal muscle stem cells. Satellite cell behavior is tightly regulated by the surrounding microenvironment, which provides multiple signals derived from local cells and systemic factors. Among epigenetic mechanisms, histone deacetylation has been proved to affect muscle regeneration. Indeed, panhistone deacetylase inhibitors were found to improve muscle regeneration, while deletion of histone deacetylase 4 (HDAC4) in satellite cells inhibits their proliferation and differentiation, leading to compromised muscle regeneration. In this study, we delineated the HDAC4 function in adult skeletal muscle, following injury, by using a tissue-specific null mouse line. We showed that HDAC4 is crucial for skeletal muscle regeneration by mediating soluble factors that influence muscle-derived cell proliferation and differentiation. These findings add new biological functions to HDAC4 in skeletal muscle that need considering when administering histone deacetylase inhibitors.

Keywords: HDAC inhibitors, satellite cells, muscle regeneration, soluble factors, muscular dystrophies

\section{INTRODUCTION}

Skeletal muscle possesses a high capacity to regenerate and muscle regeneration has been extensively studied since the $50^{\prime}$ s. In addition to physiological demand, such as during muscle growth or upon exercise, new muscle fibers are generated in response to muscle damage following injury or in degenerative diseases. Although several types of cells, including muscle-derived cells (MDCs), contribute to skeletal muscle regeneration, a crucial role of adult muscle stem cells, i.e., satellite cells (SCs), in this process is now well established (Torrente et al., 2001; Jankowski et al., 2002).

Usually quiescent, upon proper stimulation SCs become activated, proliferate, differentiate, and fuse to repair damaged myofibers or to create newly formed ones (Dumont et al., 2015). SC expansion, commitment, differentiation and fusion are sequential phases, strictly controlled by numerous transcription factors, also considered as specific stage-markers of SCs. Quiescent and activated SCs do express Pax7, a paired box transcription factor (Le Grand and Rudnicki, 2007; Wang and Rudnicki, 2012). Upon activation, SCs proliferate and can either generate selfrenewing stem cells or differentiated ones, depending on symmetric or asymmetric division (Kuang et al., 2007). Once SCs are committed to myogenesis, they start expressing other transcription factors, such as Myf5 or MyoD (Dumont et al., 2015). The transition from myoblast to myotube is mainly regulated by $\mathrm{MyoD}$, which directly regulates the transcription of the other myogenic regulatory factor (MRF) family members, myogenin and MRF4. In addition, myocyte enhancer factor-2 (Mef2) proteins co-operate with MRFs to activate the expression of skeletal muscle 
terminal differentiation genes, such as myosin heavy chains (MHCs) or creatine kinase (Lilly et al., 1994; Black and Olson, 1998) Myocytes fusion requires the expression of specific genes, including myomaker and myomerger (Millay et al., 2013; Quinn et al., 2017).

In adult skeletal myofibers, SCs reside between the basal lamina and the sarcolemma, in a surrounding microenvironment referred to as "niche." SC niche consists of the basement membrane, extracellular matrix, vascular and neural networks, several types of surrounding and interstitial cells and several diffusible molecules (Yin et al., 2013; Furuichi and Fujii, 2017). Structural and biochemical cues from the niche act on SC behavior to regulate cell quiescence, self-renewal, proliferation or differentiation, through cell-cell interactions or paracrine signals. Soluble factors play a critical role in the maintenance of muscle homeostasis in physiological conditions and may be involved in the progression of skeletal muscle diseases (De Pasquale et al., 2012; De Paepe and De Bleecker, 2013; Furuichi and Fujii, 2017). An increasing number of diffusible molecules secreted by skeletal muscle have been discovered playing a role in SC behavior. Among them, fibroblast growth factor (FGF), transforming growth factor-beta (TGF- $\beta$ ), tumor necrosis factoralpha (TNF- $\alpha$ ) or obestatin modulate different phases of muscle regeneration, in both physiological and pathological conditions (Floss et al., 1997; Coletti et al., 2005; Gurriarán-Rodríguez et al., 2015; Delaney et al., 2017; Pawlikowski et al., 2017). Also, circulating hormones regulate SC pool and skeletal muscle regeneration (Moresi et al., 2009; Costa et al., 2014; Kim et al., 2016).

Several epigenetic mechanisms are active at multiple steps of muscle regeneration, fine-tuning SC gene expression (Giordani and Puri, 2013; Moresi et al., 2015). Proper chromatin remodeling accompanies SC activation and differentiation. A permissive chromatin state that characterizes and distinguishes the pluripotency of stem cells is established by the general lack of repressive mark trimethylation of lysine 27 on histone H3 (H3K27me3) and the concomitant presence of the permissive mark trimethylation of lysine 4 on histone $\mathrm{H} 3$ (H3K4me3) at the transcription start sites (Dilworth and Blais, 2011; Moresi et al., 2015). For instance, Pax7 expression is progressively silenced during SC differentiation, changing its chromatin status from a transcriptionally permissive state, characterized by elevated levels of $\mathrm{H} 3 \mathrm{~K} 4 \mathrm{me} 3$, to a repressive one, enriched in $\mathrm{H} 3 \mathrm{~K} 27 \mathrm{me} 3$. In addition to histone methylation, histone acetyltransferases (HATs) and histone deacetylases (HDACs) modify the acetylation status of histones or transcription factors, thereby acting as transcriptional activators or repressors, respectively (Peserico and Simone, 2011). Among HDAC family members, class I HDACs inhibit MyoD gene transcription and activity. During SC differentiation, the sequential interaction of class I HDACs with MyoD and the hypo-phosphorylated pRb complex allows the transcriptional activation of the differentiation genes, such as myogenin or muscle creatine kinase (Puri et al., 2001). Instead, the class II HDAC member HDAC4 promotes SC proliferation, by repressing the transcription of the cell cycle inhibitor Cdkn1a, and differentiation, by inhibiting the expression of Sharp1 gene (Marroncelli et al., 2018). Moreover, the class II HDACs translocate from the nucleus into the cytoplasm, thereby releasing the inhibition on Mef2 and their target genes ( $\mathrm{Lu}$ et al., 2000).

Muscle regeneration: (i) relies on SCs, which are influenced by their "niche"; (ii) takes place in sequential stages, each one strictly regulated by cell-autonomous and non-autonomous cues; (iii) HDAC4 function in SCs has been partially elucidated. Therefore, in this study, we aimed to define the HDAC4 functions in adult skeletal muscle, by analyzing muscle regeneration in vivo in mice carrying a tissue-specific deletion of HDAC4.

With this purpose, we studied muscle regeneration in a mouse model in which HDAC4 was deleted specifically in skeletal muscle (HDAC4 ${ }^{\mathrm{fl} / \mathrm{fl}}$ myogenin;Cre mice, thereafter named HDAC4 mKO mice). Here we report that HDAC4 in skeletal muscle is required for proper timing and efficiency of muscle regeneration, besides HDAC4 functions in SCs. Indeed, deletion of HDAC4 compromises muscle regeneration process in vivo. MDCs from HDAC4mKO mice efficiently proliferate and differentiate in vitro, suggesting that HDAC4 mediates muscle regeneration in vivo, via soluble factors. Indeed, sera from injured HDAC4 mKO mice inhibit proliferation and differentiation of HDAC4 ${ }^{\mathrm{fl} / \mathrm{fl}} \mathrm{MDCs}$, highlighting the multiple HDAC4 functions in muscle regeneration.

\section{MATERIALS AND METHODS}

\section{Animals}

Mice were treated in strict accordance with the guidelines of the Institutional Animal Care and Use Committee, and to relevant national and European legislation, throughout the experiments. Animal protocols were approved by the Italian Ministry of Health (authorization \# 244/2013-B). HDAC4 $4^{\mathrm{fl} / \mathrm{fl}}$ myogenin-Cre (HDAC4 mKO) mice were generated by crossing $\mathrm{HDAC} 4^{\mathrm{fl} / \mathrm{fl}}$ with a mouse line expressing the Cre recombinase under the control of myogenin promoter, i.e., myogenin-Cre mice. $\mathrm{HDAC} 4^{\mathrm{fl} / \mathrm{fl}}$ mice were used as controls.

\section{Freeze Injury}

Freeze injury was performed in 8-week-old $\mathrm{HDAC}^{\mathrm{fl} / \mathrm{fl}}$ and HDAC4 mKO male mice. Mice were anesthetized with intraperitoneal (IP) injections of $50 \mathrm{mg} / \mathrm{kg}$ Zoletyl, $10 \mathrm{mg} / \mathrm{kg}$ Xylazine solution. To induce freeze injury, the tip of a steel probe precooled in dry ice was applied, for 10 seconds, to posterior muscles of anesthetized mice. This procedure induces a focal, reproducible, injury (Moresi et al., 2008; Marroncelli et al., 2018).

\section{Serum Collection}

The sera were collected by cardiac puncture in the right atrium of anesthetized male mice, 4 days after injury. About $1 \mathrm{ml}$ of blood was transferred to an Eppendorf tube and allowed to stand at room temperature for $30 \mathrm{~min}$. Blood was centrifuged at $3000 \mathrm{rpm}$ for $10 \mathrm{~min}$ and the supernatant serum was recovered. Samples were stored at $4^{\circ} \mathrm{C}$ for up to 6 months. 


\section{Histological Analyses}

Tibialis anterior muscles were dissected and embedded in Jung tissue freezing medium (Leica, Wetzlar, Germany) and frozen in liquid nitrogen precooled isopentane. Cryosections of $8 \mu \mathrm{m}$ were obtained by using a Leica cryostat. Muscles were sectioned throughout the entire length, and the injury/regeneration site was identified by extemporary toluidine blue staining. Histological sections were collected at the level of the injury/regeneration site. The section containing the maximal area of injury/regeneration was identified and further analyzed. For histological analyses, cryosections were fixed in $4 \%$ paraformaldehyde (PFA) buffered solution and stained with hematoxylin/eosin using standard method. The sections were examined with an Axioskop 2 plus system (Zeiss) microscope with relative camera AxioCamHRc and software.

\section{Morphometric Analyses}

Photomicrographs of regenerating muscles were taken at standard resolution $(1300 \times 1030$ pixel $)$ and the cross-sectional area of regenerating fibers, identified by morphological criteria (presence of centrally located nuclei) was measured by using Image J, Scion Image software. Due to the well-known wide distribution of CSAs of fibers in a regenerating muscle, we used the median of the CSA measurements to characterize our population. In addition, regenerating fiber distribution was generated by clustering the regenerating fibers into classes and expressing the value as percentage, over the regenerating fiber number.

\section{Muscle Derived Cell Isolation}

Muscle-derived cells were isolated from hind-limb muscles of 3-week-old male mice by sequential enzymatic digestions: firstly, for $30 \mathrm{~min}$ at $37^{\circ} \mathrm{C}$, with freshly prepared $1 \mathrm{mg} / \mathrm{ml}$ collagenase/dispase (Roche Diagnostics, Mannheim, Germany) in phosphate-buffered saline (PBS), followed by a second one, for $15 \mathrm{~min}$ at $37^{\circ} \mathrm{C}$, with $0.1 \mathrm{mg} / \mathrm{ml}$ type II collagenase (Sigma-Aldrich) in PBS. The enzymatic reaction was blocked by adding cell growth medium, then the cell suspension was filtered through 40-micron cell strainer filter (Falcon) and mildly centrifuged. Cells were re-suspended in growth medium and, after two preplatings of $1 \mathrm{~h}$ each to deplete fibroblasts, MDCs were plated on $0.01 \%$ collagen (Sigma-Aldrich)-coated dishes.

\section{Culture Conditions and Treatments}

Cells were cultured with Dulbecco's modified Eagle medium supplemented with $20 \%$ horse serum (Sigma-Aldrich), $100 \mathrm{U} / \mathrm{ml}$ penicillin (Sigma-Aldrich), $100 \mu \mathrm{g} / \mathrm{ml}$ streptomycin (SigmaAldrich), $50 \mu \mathrm{g} / \mathrm{ml}$ gentamicin (Sigma-Aldrich), 3\% of chicken embryo extract as growing medium (GM). After 3 days, or when cells reached $50 \%$ of confluence, GM was replaced with a differentiation medium (DM), consisting of a 1:10 dilution of GM. For conditioned cultures, horse serum was replaced with murine serum, derived from HDAC4 mKO or HDAC4 $4^{\mathrm{ll} / \mathrm{f}}$ injured mice, 4 days after the surgical procedure.

\section{Immunostaining Analyses}

For MHC immunofluorescence, differentiated cells were fixed in 4\% PFA buffered solution for $10 \mathrm{~min}$ and then blocked in 10\% goat serum in PBS for $1 \mathrm{~h}$. Cells were incubated overnight with sarcomeric MHC antibody (clone MF 20, Developmental Studies Hybridoma Bank), diluted 1:10 in 1\% BSA PBS. Fluorescent conjugated secondary anti-mouse IgG1 antibody (Alexa488, Invitrogen) diluted 1:500 in 1\% BSA PBS was used to detect the primary antibody. For Ki-67 immunofluorescence, growing cells at $24 \mathrm{~h}$ were fixed in 4\% PFA buffered solution for $10 \mathrm{~min}$, permeabilized in $0.2 \%$ Triton in PBS for $30 \mathrm{~min}$, then blocked in 3\% BSA in PBS for $20 \mathrm{~min}$. Cells were incubated overnight with 1:100 Ki-67 antibody (Santa Cruz Biotechnology) in $0.5 \%$ BSA in PBS. Fluorescent conjugated secondary anti-goat antibody (Alexa 555, Invitrogen) diluted $1: 200$ in $0.5 \%$ BSA in PBS was used to detect the primary antibody. Nuclei were counterstained with $[0.5 \mu \mathrm{g} / \mathrm{ml}]$ Hoechst and samples were mounted with $60 \%$ glycerol in Tris $\mathrm{HCl} 0.2 \mathrm{M}$ pH 9.3.

\section{RT-PCR and Real-Time PCR}

Total RNA from Tibialis Anterior muscle was isolated using TRIzoL reagent (Thermo Fisher), according to manufacturer's instructions, 4 days following injury. cDNA synthesis was performed from 0.5 to $1 \mu \mathrm{g}$ of RNA using Reverse Transcription Kit (Takara), following the manufacturer's instructions. Quantitative PCR was performed using the ABI PRISM 700 SDS (Applied Biosystems) with SYBR Green reagent (Applied Biosystems) and primer listed in Table $\mathbf{1 .}$

\section{Statistics}

Statistical significance was determined using two-tailed Student's $t$-test with a significance level $<0.05$, or by using one-way analysis of variance (ANOVA), followed by Tukey's HSD as a post hoc test, when more than two conditions needed to be compared. All values are expressed as mean \pm standard error of the mean (SEM). VassarStats, a statistical computation website

TABLE 1 | Primers used for real-time PCR.

\begin{tabular}{llll}
\hline Gene & Reference number & \multicolumn{1}{c}{ Forward primer } & Reverse primer \\
\hline HDAC4 & NM_207225.2 & GTCTTGGGAATGTACGACGC & GTTGCCAGAGCTGCTATTG \\
Pax7 & NM_011039.2 & TCCCCCTGGAAGTGTCCA & TGGGAACACGGAGCTGA \\
MyoD & M84918.1 & ACCCAGGAACTGGGTGGA & AAGTCGTCTGCTGTCTCAAA \\
Myogenin & NM_031189.2 & GCACTGGAGTCGGTCCCAA & TATCCTCCACCGTGATGCTG \\
e-MHC & M11154.1 & TGGTCGTAATCAGCAGCA & TCGTCTCGCTTGGCAA
\end{tabular}


available at http://vassarstats.net/, was used for the statistical analyses.

\section{RESULTS}

\section{HDAC4 Expression Is Modulated in Skeletal Muscle Upon Injury}

Aiming to investigate the role of HDAC4 in skeletal muscle regeneration, we evaluated its expression levels in regenerating skeletal muscle over time. The tibialis anterior muscle of adult $\mathrm{HDAC} 4^{\mathrm{fl} / \mathrm{fl}}$ male mice was subjected to a localized, reproducible, freeze injury to induce regeneration, (Moresi et al., 2008) and HDAC4 expression was evaluated over time, by real-time PCR (Figure 1). HDAC4 expression in skeletal muscle is significantly induced upon injury, compared to un-injured muscles, and reached a peak of expression at day 4 , suggesting a role for this epigenetic factor in the early phases of muscle regeneration.

\section{Deletion of HDAC4 in Skeletal Muscle Compromised Muscle Regeneration}

The expression of HDAC4 is significantly up-regulated in skeletal muscle in response to injury. Therefore, to define HDAC4 functions in adult skeletal muscle, we analyzed muscle regeneration in a mouse line in which HDAC4 deletion is mediated by a Cre-recombinase under the control of the myogenin promoter, i.e., since the embryonic stage E8.5 (HDAC4 mKO mice) (Cheng et al., 1992). This mouse line does not show overt abnormalities in skeletal muscle under physiological conditions (Moresi et al., 2010; Pigna et al., 2018). However, 1 week after injury, HDAC4 mKO mice showed smaller regenerating fibers than $\mathrm{HDAC}_{4}^{\mathrm{fl} / \mathrm{fl}}$ mice, used as controls, by histological analyses (Figure 2A). Morphometric analyses of regenerating myofiber cross-sectional area (CSA)

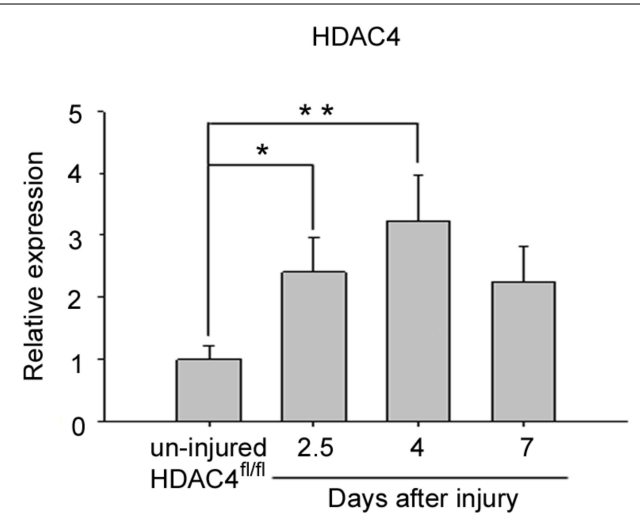

FIGURE 1 | HDAC4 expression is up-regulated in regenerating HDAC4 4 fl/fl muscles. Real-time PCR for HDAC4 in regenerating muscles, at the indicated time points following injury, over un-injured muscles. Data are presented as mean + /- SEM. $n=6$ mice for each condition. One-way ANOVA showed a significant effect of the treatment between groups $(F=5.16 ; d f=3 ; p=0.01$ ) and a significant interaction between un-injured $\mathrm{HDAC}^{\mathrm{fl} / \mathrm{fl}}$ mice and 2.5 days $\left({ }^{*} p<0.05\right)$ or 4 days $\left({ }^{* *} p<0.01\right)$ after injury by Tukey's HSD test. confirmed that HDAC4 mKO mice exhibited smaller, centrally nucleated myofibers with respect to $\mathrm{HDAC}^{\mathrm{fl} / \mathrm{fl}}$ mice (Figure $2 \mathbf{B}$ ). Regenerating fiber distribution confirmed that HDAC4 KO mice displayed a significantly higher number of smaller regenerating myofibers $\left(400-599 \mu \mathrm{m}^{2}\right)$ than $\mathrm{HDAC}^{\mathrm{fl} / \mathrm{fl}}$ mice, at the expenses of the larger ones (1000-1199 $\mu \mathrm{m}^{2}$ ) (Figure 2C). Molecular analyses performed at the time of maximal expression of HDAC4 in regenerating muscles, i.e., 4 days following injury, corroborated a significant reduction of the expression of myogenic markers of early, intermediate and terminal differentiation, i.e., Pax7, MyoD, myogenin and embryonic $\mathrm{MHC}$, in HDAC4 $\mathrm{mKO}$ mice, compared to $\mathrm{HDAC} 4^{\mathrm{fl} / \mathrm{fl}}$ mice (Figure 2D).

To discriminate whether the HDAC4 deletion in skeletal muscle resulted in delayed or compromised muscle regeneration, we analyzed regenerating muscles at later time points, 2 weeks and 1 month after injury. Differences in muscle regeneration ability persisted between genotypes after 2 weeks and after 1 month following injury, as shown by histological and morphometrical analyses of regenerating fiber CSA (Figure 3), indicating that deletion of HDAC4 in skeletal muscle is sufficient to hamper muscle regeneration.

\section{HDAC4 mKO Muscle Derived Cells Efficiently Proliferate and Differentiate in vitro}

Because HDAC4 is crucial for muscle stem cell proliferation and differentiation (Marroncelli et al., 2018), we wondered whether MDC proliferation and/or differentiation was affected in HDAC4 mKO mice. MDCs were isolated, and cell proliferation was assessed by immunofluorescence for Ki-67, a protein associated with cellular proliferation, after $24 \mathrm{~h}$ in growing condition. HDAC4 mKO MDCs showed a similar amount of Ki-67 positive cells, and of total MDCs, respect to $\mathrm{HDAC} 4^{\mathrm{fl} / \mathrm{fl}}$ mice (Figures 4A-C). Terminal differentiation was induced and assessed by immunofluorescence for MHC (Figure 4D). No significant differences in terminal differentiation were detected between HDAC4 $\mathrm{mKO}$ and HDAC4 ${ }^{\mathrm{fl} / \mathrm{fl}} \mathrm{MDCs}$, as also quantified by the differentiation (i.e., the number MHC positive cells, over total nuclei) or the fusion (the number of myonuclei in myotubes, over total nuclei) indexes (Figure 4E).

These data indicate that deletion of HDAC4, upon myogenin expression, does not affect MDC proliferation or differentiation, implying that $\mathrm{HDAC} 4$ controls muscle regeneration in vivo via soluble factors.

\section{HDAC4 mKO Serum Negatively Affects MDC Proliferation and Differentiation}

To prove that HDAC4 affects MDC biology in a cell nonautonomous manner, HDAC4 $\mathrm{mKO}$ and $\mathrm{HDAC}^{\mathrm{fl} / \mathrm{fl}}$ sera were withdrawn 4 days following injury, at the time of the HDAC4 maximal expression in skeletal muscle. Control MDCs were cultured with conditioned media by using HDAC4 $\mathrm{mKO}$ or $\mathrm{HDAC}^{\mathrm{fl} / \mathrm{fl}}$ sera, and MDC proliferation was evaluated after $24 \mathrm{~h}$ in growing condition, by $\mathrm{Ki}-67$ immunofluorescence (Figure 5A). Quantification of Ki-67-positive cells revealed that 
A

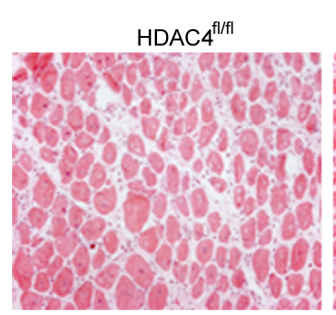

C

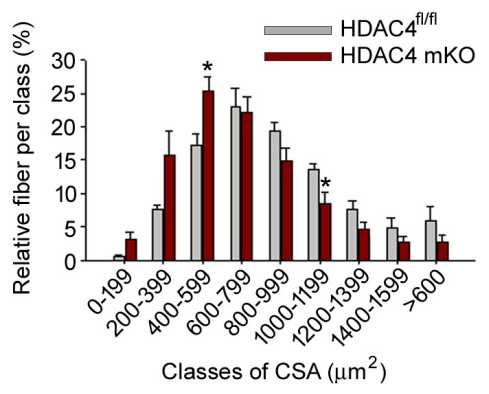

D

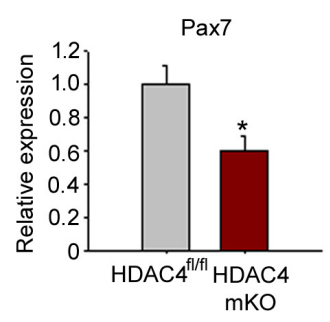

HDAC4 mKO

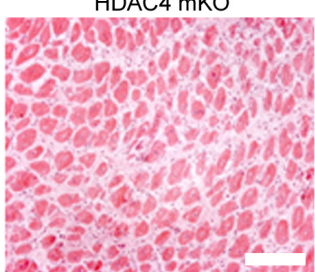

B

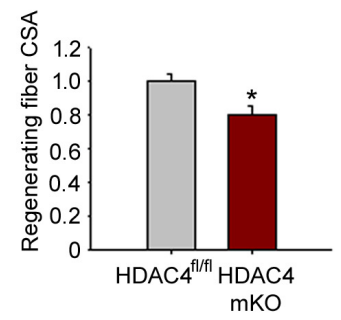

FIGURE 2 | HDAC4 mKO mice exhibit delayed muscle regeneration. (A) Representative images of HDAC4 mKO and HDAC4 ${ }^{f / f l}$ tibialis anterior regenerating muscles, 1 week after injury. Scale bar $=50$ micron. (B) Regenerating fiber CSA of HDAC4 mKO and HDAC4 ${ }^{\mathrm{fl} / \mathrm{fl}}$ mice, 1 week after injury. Data are presented as median $+/$ - SEM. $n=8$ mice for genotype. ${ }^{*} p<0.05$ by Student's $t$-test. (C) Morphometric analysis of the distribution of regenerating fiber cross-sectional area, 1 week after injury. $n=8$ mice for genotype. Data are presented as average +/- SEM. ${ }^{*} p<0.05$ by Student's $t$-test. (D) Gene expression of indicated myogenic markers in HDAC4 mKO and HDAC4 ${ }^{\mathrm{fl} / \mathrm{fl}}$ mice, by real-time PCR, 4 days following injury. Data are presented as mean $+/-\mathrm{SEM} . n=8$ mice for genotype. ${ }^{*} p<0.05$; ${ }^{* *} p<0.005$ by Student's $t$-test.

HDAC4 $\mathrm{mKO}$ sera reduced the MDC proliferating cell number, compared to HDAC4 $4^{\mathrm{fl} / \mathrm{fl}}$ sera (Figure 5B), data also confirmed by the quantification of the MDCs (Figure 5C).

To investigate if the reduction in MDC proliferation influences their terminal differentiation, control MDC were induced to differentiate, by mean of conditioned media with HDAC4 mKO or HDAC4 $4^{\mathrm{f} / \mathrm{fl}}$ mouse sera, as control. HDAC4 mKO sera significantly reduced also MDC terminal differentiation and fusion, as assessed by immunofluorescence for MHC and quantification of the differentiation and fusion indexes (Figures 5D,E). Quantification of MDCs, after 3 days of differentiation, confirmed a significant reduction in the MDC number upon HDAC4 mKO serum treatment with respect to controls (Figure 5F). These data demonstrate that HDAC4 mediates the production and release of circulating factors able to negatively affect MDC proliferation and differentiation.

\section{DISCUSSION}

Numerous studies clarified that MDC biology is controlled by cell-autonomous and non-autonomous cues, as well as by epigenetic mechanisms (Boonen and Post, 2008; Moresi et al., 2015). In this study, we investigated which functions HDAC4 plays during muscle regeneration. HDAC4 is a stress-responsive epigenetic factor known to regulate multiple responses in skeletal muscle, including satellite cells biology upon injury (Moresi et al., 2010; Choi et al., 2014; Marroncelli et al., 2018). We found HDAC4 to be induced to a significant extent in skeletal muscle upon injury, in line with previous findings (Choi et al., 2014), with the expression peaking in the early phases of regeneration, suggesting that HDAC4 mediates skeletal muscle response to injury. To dissect the HDAC4 biological functions in skeletal muscle, excluding SCs in the early phases of differentiation, we generated HDAC4 mKO mice, in which HDAC4 deletion occurs upon myogenin expression. HDAC4 mKO mice do not show skeletal muscle abnormalities at baseline (Moresi et al., 2010; Pigna et al., 2018). However, following injury, deletion of HDAC4 in skeletal muscle significantly hampered muscle regeneration. When HDAC4 deletion occurs in myogenin-positive cells, MDCs efficiently proliferate and differentiate in vitro, differently from HDAC4 KO SCs, having compromised expansion and differentiation in a cell-autonomous manner (Choi et al., 2014; Marroncelli 
A

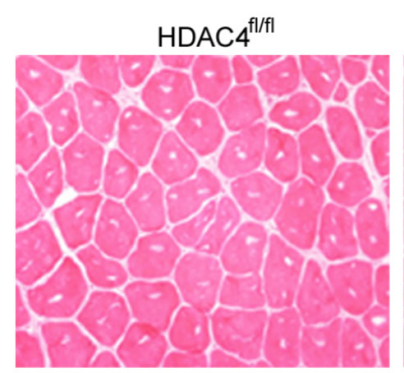

C

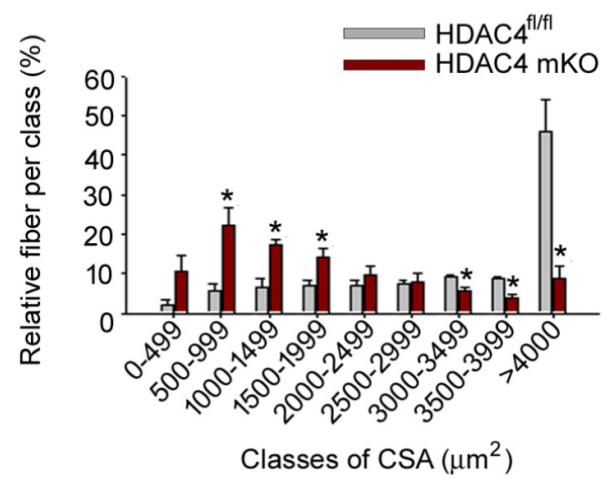

D
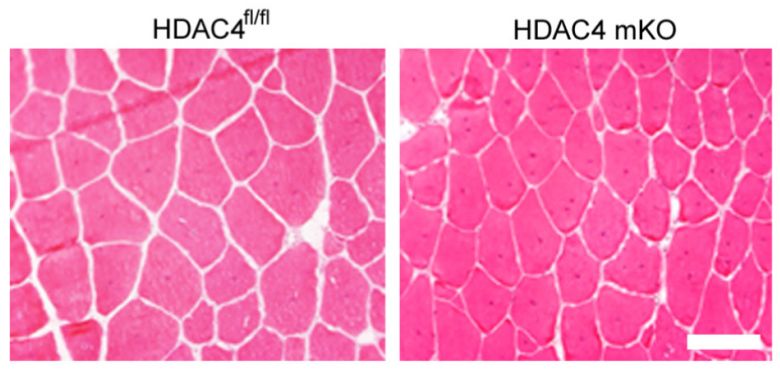

$\mathbf{F}$

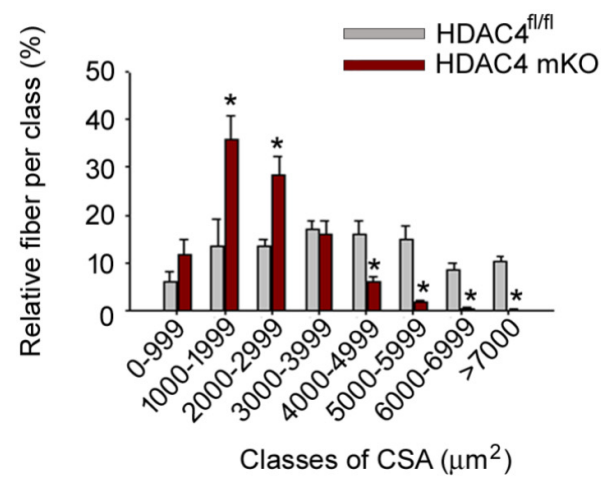

B

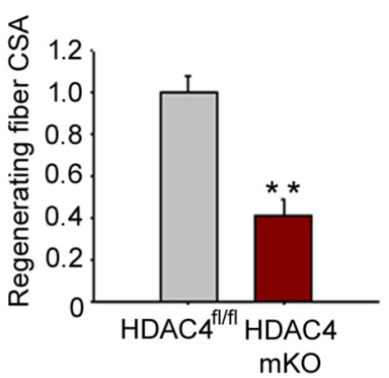

E

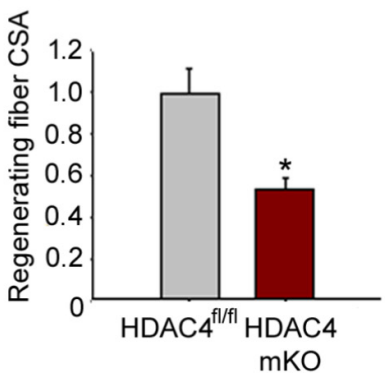

FIGURE 3 | HDAC4 mKO mice show impaired muscle regeneration. (A) Representative images of HDAC4 mKO and HDAC4 $4^{\mathrm{fl} / \mathrm{fl}}$ tibialis anterior regenerating muscles, 2 weeks after injury. Scale bar $=50$ micron. (B) Regenerating fiber CSA of HDAC4 mKO and HDAC4 $4^{\mathrm{f} / \mathrm{fl}}$ mice, 2 weeks after injury. Data are presented as median $+/-$ SEM. $n=8$ mice for genotype. ${ }^{* *} p<0.02$ by Student's $t$-test. (C) Morphometric analysis of the distribution of regenerating fiber cross-sectional area, 2 weeks after injury. $n=8$ mice for genotype. Data are presented as average + -- SEM. ${ }^{*} p<0.05$ by Student's $t$-test. (D) Representative images of HDAC4 mKO and $\mathrm{HDAC}^{\mathrm{fl} / \mathrm{fl}}$ tibialis anterior regenerating muscles, 1 month after injury. Scale bar $=50$ micron. (E) Regenerating fiber CSA of HDAC4 mKO and HDAC4 ${ }^{f / / f l}$ mice, 1 month after injury. $n=8$ mice for genotype. Data are presented as median $+/-$ SEM. ${ }^{*} p<0.05$ by Student's $t$-test. (F) Morphometric analysis of the distribution of regenerating fiber cross-sectional area, 1 month after injury. $n=5$ mice for genotype. Data are presented as average $+/-$ SEM. * $p<0.05$ by Student's $t$-test.

et al., 2018). Despite MDC ability to differentiate in vitro, compromised muscle regeneration in HDAC4 mKO mice indicates that, in the injured muscle, HDAC4 influences MDCs behavior, evidencing novel HDAC4 cellular functions depending on the timing and/or the cell sub-type where this enzyme is expressed. 


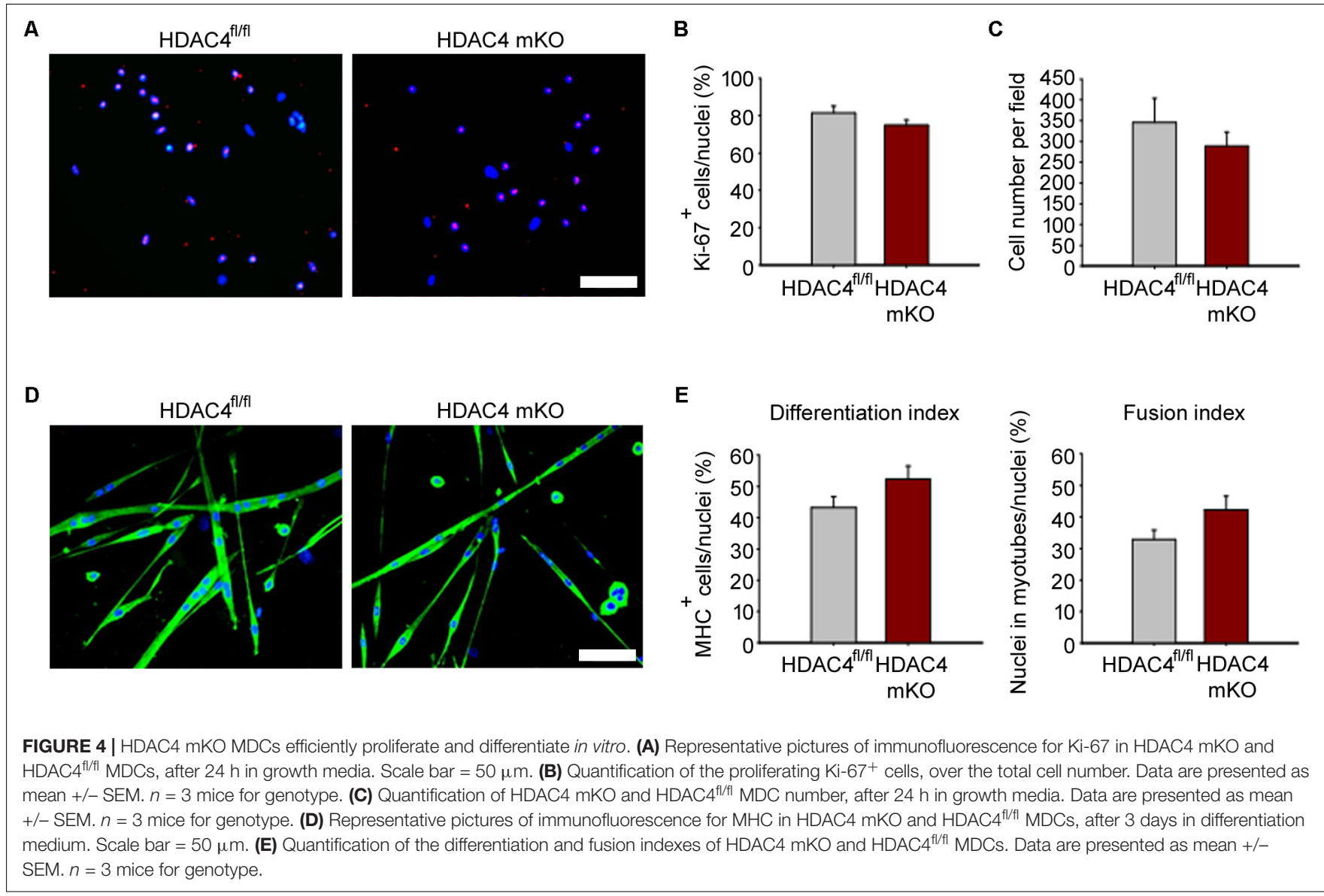

Interestingly, the decrease of the expression level of the SCs marker Pax7 4 days after injury indicates a deficit in either SC number or proliferation. The paired box transcription factor Pax7 is the master regulator of SC (Seale et al., 2000), required for SC function in adult skeletal muscle. Indeed, upon Pax7 deletion, SCs exhibit cell-cycle arrest and dysregulation of MRFs, muscles resulted smaller and muscle regeneration was severely impaired (Oustanina et al., 2004; Kuang et al., 2006; Relaix et al., 2006). Since HDAC4 mKO MDCs were able to proliferate or differentiate efficiently in vitro, soluble factors likely mediate the reduction of Pax7 expression in regenerating HDAC4 mKO muscles.

By using culture media conditioned with sera from injured HDAC4 $\mathrm{mKO}$ or $\mathrm{HDAC}_{4}^{\mathrm{fl} / \mathrm{fl}}$ mice, we proved that HDAC4 does mediate the secretion of circulating factors able to influence MDC proliferation and differentiation. Numerous environmental factors regulate adult myogenesis during regeneration. Growth factors released from injured myofibers strictly regulate the activation of quiescent SC (Furuichi and Fujii, 2017). SC proliferation is supported by mitogens such as FGF and insulinlike growth factor (IGF), which get up-regulated in skeletal muscle after injury (Guthridge et al., 1992; Bischoff and Heintz, 1994). Furthermore, activated SCs secrete miRNAs - containing exosomes, which in turn modulate SC proliferation and differentiation (Harding and Velleman, 2016). SC differentiation is strictly influenced by secreted factors as well. For instance, insulin-like growth factor 1 (IGF1) and interleukin 15 (IL-15) secretion is induced after membrane damage or during exercise, these myokines promote SC differentiation and contributes to muscle hypertrophy by enhancing protein synthesis (DeVol et al., 1990; Adams, 2002; Quinn et al., 2002; Furmanczyk and Quinn, 2003). Among soluble factors acting on SC differentiation, several inflammatory cytokines are known to inhibit this process. TNF- $\alpha$ and interleukin 1 (IL-1) are inflammatory mediators of muscle wasting and interfere with the expression of myogenic factors in differentiating myoblasts, by activating the nuclear factor - kappa beta $(\mathrm{NFk} \beta)$ and caspases (Langen et al., 2001; Moresi et al., 2008, 2009). In addition to inhibit SC activation and self-renewal, numerous secreted factors negatively affect also SC differentiation and fusion, among them myostatin and growth differentiation factor11 (GDF11) (TGFß superfamily's members) are known to hamper muscle regeneration by inhibiting SC activity on distinct phases during myogenesis (Reisz-Porszasz et al., 2003; Egerman et al., 2015).

HDACs have been shown to regulate soluble factors in different cell types. In neuronal and glial cells, the release of brain-derived neurotrophic factor (BDNF) and fibroblast growth factor 1 (FGF1) is mediated by HDACs (Hossain 
A

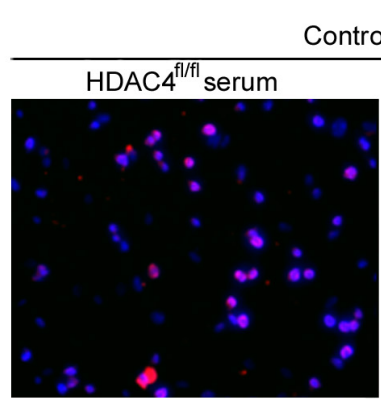

D

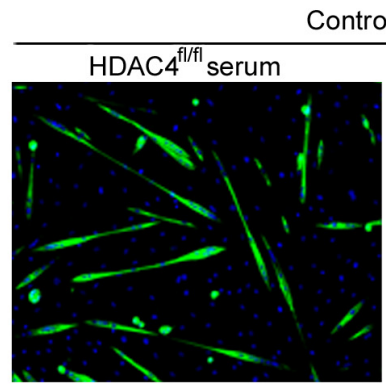

E

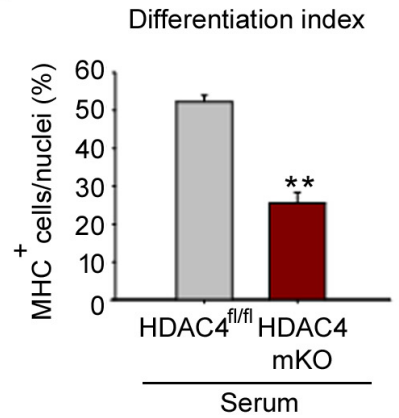

B

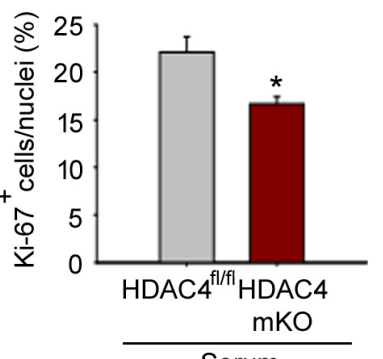

Serum
C

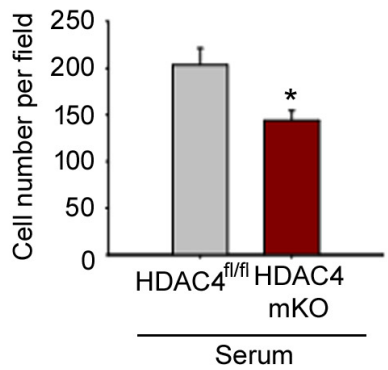

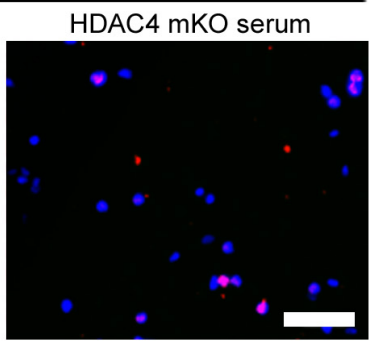

ontrol MDC

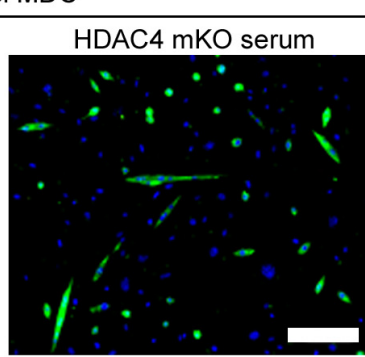

FIGURE 5 | HDAC4 mKO serum negatively affects control MDC proliferation and differentiation. (A) Representative pictures of immunofluorescence for Ki-67 in control MDCs, after $24 \mathrm{~h}$ in conditioned media with sera from either injured HDAC4 mKO or HDAC4l/fl mice. Scale bar $=50 \mu \mathrm{m}$. (B) Quantification of the proliferating $\mathrm{Ki}-67^{+}$cells, over the total cell number. Data are presented as mean $+/-$SEM. $n=6$ mice for genotype. * $p<0.05$ by Student's $t$-test. (C) Quantification of MDC number cultured with sera from either injured HDAC4 mKO or HDAC4 ${ }^{\mathrm{fl} / \mathrm{fl}}$ mice. Data are presented as mean $+/-\mathrm{SEM}$. $n=6$ mice for genotype. * $p<0.05$ by Student's t-test. (D) Representative pictures of immunofluorescence for MHC in control MDCs cultured with conditioned media with sera from either HDAC4 mKO or HDAC4 $^{\mathrm{fl} / \mathrm{fl}}$ injured mice, after 3 days in differentiation medium. Scale bar $=100 \mu \mathrm{m}$. (E) Quantification of the differentiation and fusion indexes of control MDCs cultured with sera from either injured HDAC4 mKO or HDAC4 $4^{\mathrm{fl} / \mathrm{fl}}$ mice, after 3 days in differentiation medium. Data are presented as mean $+/-$ SEM. $n=6$ mice for genotype. ${ }^{* *} p<0.005$ by Student's $t$-test. (F) Number of control MDCs cultured with sera from either injured HDAC4 mKO or HDAC4 ${ }^{\text {fl/fl }}$ mice, after 3 days in differentiation medium. Data are presented as mean +/- SEM. $n=6$ mice for genotype. ** $p<0.005$ by Student's $t$-test.

et al., 2018), as well as in fibroblasts the production of several proinflammatory cytokines/chemokines (Li et al., 2011), thus contributing to chronic inflammatory processes. Moreover, HDACs modulate IL-4 expression and secretion in mast cells and monocyte-derived DCs (moDCs) (López-Bravo et al., 2013; Nakamaru et al., 2015). In addition to its classical roles, new evidence links HDACs and soluble factors. For instance, cytoplasmic protein acetylation/deacetylation balance has been involved in extracellular vesicles content and release in colon cancer cells (Li et al., 2016; Chao et al., 2017). In skeletal muscle, the expression of several soluble factors, such as TGF- $\beta$ and follistatin, are modulated by HDAC4 during myogenesis (Sun et al., 2010; Winbanks et al., 2011). Moreover, HDAC4 promotes skeletal muscle reinnervation via the release of FGF binding protein 1 (Williams et al., 2009).

A preclinical study in a murine Duchenne Muscular Dystrophy model demonstrated that Givinostat, a class I and 
II HDAC inhibitor, improves skeletal muscle regeneration by reducing fibrotic and adipose tissues (Mozzetta et al., 2013). Similarly, trichostatin A (TSA), another class I-II HDAC inhibitor, promotes skeletal muscle regeneration by targeting fibro-adipogenic cells and inducing the expression and the release of follistatin, a pro-myogenic soluble factor identified as a central mediator in myoblast recruitment and fusion (Iezzi et al., 2004; Mozzetta et al., 2013). Our results are in apparent contrast with the findings of previous studies obtained by administering HDAC inhibitors during muscle regeneration. However, different experimental conditions could reconcile such discrepancy. In those studies, muscle regeneration was improved by daily systemic administration of class I and II HDAC inhibitors after injury, which is different from our study exploiting HDAC4 mKO mice, a tissue-specific KO mouse bringing the genetic deletion at embryonic stage E8.5 of one member of the class IIa HDACs.

\section{CONCLUSION}

The present study sheds further light on multiple HDAC4 functions in skeletal muscle following injury stress. HDAC4 not only promotes SC replenishment and differentiation in a cell-autonomous manner via the epigenetic regulation of gene transcription but also influences MDC proliferation and differentiation via muscle derived-soluble factors. These findings should be considered when administering class I-II HDAC inhibitors to treat muscular diseases.

\section{REFERENCES}

Adams, G. R. (2002). Invited review: autocrine/paracrine IGF-I and skeletal muscle adaptation. J. Appl. Physiol. 93, 1159-1167. doi: 10.1152/japplphysiol.01264. 2001

Bischoff, R., and Heintz, C. (1994). Enhancement of skeletal muscle regeneration. Dev. Dyn. 201, 41-54. doi: 10.1002/aja.1002010105

Black, B. L., and Olson, E. N. (1998). Transcriptional control of muscle development by myocyte enhancer factor-2 (mef2) proteins. Annu. Rev. Cell Dev. Biol. 14, 167-196. doi: 10.1146/annurev.cellbio.14.1.167

Boonen, K. J. M., and Post, M. J. (2008). The muscle stem cell niche: regulation of satellite cells during regeneration. Tissue Eng. B Rev. 14, 419-431. doi: 10.1089/ ten.teb.2008.0045

Chao, O. S., Chang, T. C., Di Bella, M. A., Alessandro, R., Anzanello, F., Rappa, G., et al. (2017). The HDAC6 inhibitor tubacin induces release of CD133+extracellular vesicles from cancer cells. J. Cell. Biochem. 118, 44144424. doi: $10.1002 /$ jcb. 26095

Cheng, T.-C., Hanley, T. A., Mudd, J., Merlie, J. P., and Olson, E. N. (1992). Mapping of myogenin transcription during embryogenesis using transgenes linked to the myogenin control region. J. Cell Biol. 119, 1649-1656. doi: 10.1083/ jcb.119.6.1649

Choi, M.-C., Ryu, S., Hao, R., Wang, B., Kapur, M., Fan, C.-M., et al. (2014). HDAC4 promotes Pax7-dependent satellite cell activation and muscle regeneration. EMBO Rep. 15, 1175-1183. doi: 10.15252/embr. 201439195

Coletti, D., Moresi, V., Adamo, S., Molinaro, M., and Sassoon, D. (2005). Tumor necrosis factor- $\alpha$ gene transfer induces cachexia and inhibits muscle regeneration. Genesis 43, 120-128. doi: 10.1002/gene.20160

Costa, A., Rossi, E., Scicchitano, B. M., Coletti, D., Moresi, V., and Adamo, S. (2014). Neurohypophyseal hormones: novel actors of striated muscle development and homeostasis. Eur. J. Transl. Myol. 24:3790. doi: 10.4081/ejtm. 2014.3790

\section{AUTHOR CONTRIBUTIONS}

$\mathrm{AR}, \mathrm{NM}$, and $\mathrm{CN}$ performed the experiments. $\mathrm{VM}$ and SA conceived the project and analyzed the data. All authors contributed to critical analysis. AR, VM, and SA wrote the manuscript and all authors approved the final manuscript for publication.

\section{FUNDING}

This study has been funded by Ricerca finalizzata (GR-20102311055) from the Italian Ministry of Health and partially supported by Sapienza research projects 2016 (SapMedi2016) and 2017 (RM11715C78539BD8).

\section{ACKNOWLEDGMENTS}

We are grateful to Carla Ramina for her technical assistance. We are grateful to Prof. Eric N Olson and Rhonda Bassel-Duby (University of Texas Southwestern Medical Center, Dallas, TX, United States) for the mouse line and the scientific advice. The MF 20 monoclonal antibody was developed by D. A. Fishman and obtained from the Developmental Studies Hybridoma Bank, created by the NICHD of the NIH and maintained at the University of Iowa, Department of Biology, Iowa City, IA, United States, 52242.

De Paepe, B., and De Bleecker, J. L. (2013). Cytokines and chemokines as regulators of skeletal muscle inflammation: presenting the case of Duchenne muscular dystrophy. Mediators Inflamm. 2013:540370. doi: 10.1155/2013/ 540370

De Pasquale, L., D’Amico, A., Verardo, M., Petrini, S., Bertini, E., and De Benedetti, F. (2012). Increased muscle expression of interleukin-17 in Duchenne muscular dystrophy. Neurology 78, 1309-1314. doi: 10.1212/WNL. 0b013e3182518302

Delaney, K., Kasprzycka, P., Ciemerych, M. A., and Zimowska, M. (2017). The role of TGF- $\beta 1$ during skeletal muscle regeneration. Cell Biol. Int. 41, 706-715. doi: 10.1002/cbin.10725

DeVol, D. L., Rotwein, P., Sadow, J. L., Novakofski, J., and Bechtel, P. J. (1990). Activation of insulin-like growth factor gene expression during work-induced skeletal muscle growth. Am. J. Physiol. Metab. 259, E89-E95. doi: 10.1152/ ajpendo.1990.259.1.E89

Dilworth, F. J., and Blais, A. (2011). Epigenetic regulation of satellite cell activation during muscle regeneration. Stem Cell Res. Ther. 2:18. doi: 10.1186/ scrt59

Dumont, N. A., Bentzinger, C. F., Sincennes, M.-C., and Rudnicki, M. A. (2015). Satellite cells and skeletal muscle regeneration. Compr. Physiol. 5, 1027-1059. doi: 10.1002/cphy.c140068

Egerman, M. A., Cadena, S. M., Gilbert, J. A., Meyer, A., Nelson, H. N., Swalley, S. E., et al. (2015). GDF11 increases with age and inhibits skeletal muscle regeneration. Cell Metab. 22, 164-174. doi: 10.1016/j.cmet.2015. 05.010

Floss, T., Arnold, H. H., and Braun, T. (1997). A role for FGF-6 in skeletal muscle regeneration. Genes Dev. 11, 2040-2051. doi: 10.1101/gad.11. 16.2040

Furmanczyk, P. S., and Quinn, L. S. (2003). Interleukin15 increases myosin accretion in human skeletal myogenic cultures. Cell Biol. Int. 27, 845-851. doi: 10.1016/S1065-6995(03) 00172-0 
Furuichi, Y., and Fujii, N. L. (2017). Mechanism of satellite cell regulation by myokines. J. Phys. Fit. Sport Med. 6, 311-316. doi: 10.7600/jp fsm.6.311

Giordani, L., and Puri, P. L. (2013). Epigenetic control of skeletal muscle regeneration. FEBS J. 280, 4014-4025. doi: 10.1111/febs.12383

Gurriarán-Rodríguez, U., Santos-Zas, I., González-Sánchez, J., Beiroa, D., Moresi, V., Mosteiro, C. S., et al. (2015). Action of obestatin in skeletal muscle repair: stem cell expansion, muscle growth, and microenvironment remodeling. Mol. Ther. 23, 1003-1021. doi: 10.1038/mt.2015.40

Guthridge, M., Wilson, M., Cowling, J., Bertolini, J., and Hearn, M. T. W. (1992). The role of basic fibroblast growth factor in skeletal muscle regeneration. Growth Factors 6, 53-63. doi: 10.3109/08977199209 008871

Harding, R. L., and Velleman, S. G. (2016). MicroRNA regulation of myogenic satellite cell proliferation and differentiation. Mol. Cell. Biochem. 412, 181-195. doi: 10.1007/s11010-015-2625-6

Hossain, M. S., Oomura, Y., and Katafuchi, T. (2018). Glucose can epigenetically alter the gene expression of neurotrophic factors in the murine brain cells. Mol. Neurobiol. 55, 3408-3425. doi: 10.1007/s12035-017-0578-3

Iezzi, S., Di Padova, M., Serra, C., Caretti, G., Simone, C., Maklan, E., et al. (2004). Deacetylase inhibitors increase muscle cell size by promoting myoblast recruitment and fusion through induction of follistatin. Dev. Cell 6, 673-684. doi: 10.1016/S1534-5807(04)00107-8

Jankowski, R. J., Deasy, B. M., and Huard, J. (2002). Muscle-derived stem cells. Gene Ther. 9, 642-647. doi: 10.1038/sj.gt.3301719

Kim, J.-H., Han, G.-C., Seo, J.-Y., Park, I., Park, W., Jeong, H.-W., et al. (2016). Sex hormones establish a reserve pool of adult muscle stem cells. Nat. Cell Biol. 18, 930-940. doi: 10.1038/ncb3401

Kuang, S., Chargé, S. B., Seale, P., Huh, M., and Rudnicki, M. A. (2006). Distinct roles for Pax7 and Pax3 in adult regenerative myogenesis. J. Cell Biol. 172, 103-113. doi: 10.1083/jcb.200508001

Kuang, S., Kuroda, K., Le Grand, F., and Rudnicki, M. A. (2007). Asymmetric selfrenewal and commitment of satellite stem cells in muscle. Cell 129, 999-1010. doi: 10.1016/j.cell.2007.03.044

Langen, R. C., Schols, A. M., Kelders, M. C., Wouters, E. F., and Janssen-Heininger, Y. M. (2001). Inflammatory cytokines inhibit myogenic differentiation through activation of nuclear factor-kappaB. FASEB J. 15, 1169-1180. doi: 10.1016/j.cell. 2007.03.044

Le Grand, F., and Rudnicki, M. A. (2007). Skeletal muscle satellite cells and adult myogenesis. Curr. Opin. Cell Biol. 19, 628-633. doi: 10.1016/J.CEB.2007.09.012

Li, M., Riddle, S. R., Frid, M. G., El Kasmi, K. C., McKinsey, T. A., Sokol, R. J., et al. (2011). Emergence of fibroblasts with a proinflammatory epigenetically altered phenotype in severe hypoxic pulmonary hypertension. J. Immunol. 187, 2711-2722. doi: 10.4049/jimmunol.1100479

Li, Z., Zhuang, M., Zhang, L., Zheng, X., Yang, P., and Li, Z. (2016). Acetylation modification regulates GRP78 secretion in colon cancer cells. Sci. Rep. 6:30406. doi: $10.1038 /$ srep30406

Lilly, B., Galewsky, S., Firulli, A. B., Schulz, R. A., and Olson, E. N. (1994). D-MEF2: a MADS box transcription factor expressed in differentiating mesoderm and muscle cell lineages during Drosophila embryogenesis. Proc. Natl. Acad. Sci. U.S.A. 91, 5662-5666.

López-Bravo, M., Minguito de la Escalera, M., Domínguez, P. M., GonzálezCintado, L., del Fresno, C., Martín, P., et al. (2013). IL-4 blocks TH1polarizing/inflammatory cytokine gene expression during monocyte-derived dendritic cell differentiation through histone hypoacetylation. J. Allergy Clin. Immunol. 132, 1409.e13-1419.e13. doi: 10.1016/j.jaci.2013.08.039

Lu, J., McKinsey, T. A., Nicol, R. L., and Olson, E. N. (2000). Signal-dependent activation of the MEF2 transcription factor by dissociation from histone deacetylases. Proc. Natl. Acad. Sci. U.S.A. 97, 4070-4075. doi: 10.1073/pnas. 080064097

Marroncelli, N., Bianchi, M., Bertin, M., Consalvi, S., Saccone, V., De Bardi, M., et al. (2018). HDAC4 regulates satellite cell proliferation and differentiation by targeting P21 and Sharp1 genes. Sci. Rep. 8:3448. doi: 10.1038/s41598-01821835-7

Millay, D. P., O’Rourke, J. R., Sutherland, L. B., Bezprozvannaya, S., Shelton, J. M., Bassel-Duby, R., et al. (2013). Myomaker is a membrane activator of myoblast fusion and muscle formation. Nature 499, 301-305. doi: 10.1038/nat ure 12343
Moresi, V., Garcia-Alvarez, G., Pristerà, A., Rizzuto, E., Albertini, M. C., Rocchi, M., et al. (2009). Modulation of caspase activity regulates skeletal muscle regeneration and function in response to vasopressin and tumor necrosis factor. PLoS One 4:e5570. doi: 10.1371/journal.pone. 0005570

Moresi, V., Marroncelli, N., and Adamo, S. (2015). New insights into the epigenetic control of satellite cells. World J. Stem Cells 7, 945-955. doi: 10.4252/wjsc.v7. i6.945

Moresi, V., Pristerà, A., Scicchitano, B. M., Molinaro, M., Teodori, L., Sassoon, D., et al. (2008). Tumor necrosis factor- $\alpha$ inhibition of skeletal muscle regeneration is mediated by a caspase-dependent stem cell response. Stem Cells 26, 997-1008. doi: 10.1634/stemcells.2007-0493

Moresi, V., Williams, A. H., Meadows, E., Flynn, J. M., Potthoff, M. J., McAnally, J., et al. (2010). Myogenin and class II HDACs control neurogenic muscle atrophy by inducing E3 ubiquitin ligases. Cell 143, 35-45. doi: 10.1016/j.cell.2010. 09.004

Mozzetta, C., Consalvi, S., Saccone, V., Tierney, M., Diamantini, A., Mitchell, K. J., et al. (2013). Fibroadipogenic progenitors mediate the ability of HDAC inhibitors to promote regeneration in dystrophic muscles of young, but not old Mdx mice. EMBO Mol. Med. 5, 626-639. doi: 10.1002/emmm. 201202096

Nakamaru, Y., Takagi, D., Homma, A., Hatakeyama, S., and Fukuda, S. (2015). Oxidative stress regulates IL-4 gene expression in mast cells through the reduction of histone deacetylase. Otolaryngol. Head Neck Surg. 152, 48-52. doi: $10.1177 / 0194599814559189$

Oustanina, S., Hause, G., and Braun, T. (2004). Pax7 directs postnatal renewal and propagation of myogenic satellite cells but not their specification. EMBO J.23, 3430-3439. doi: 10.1038/sj.emboj.7600346

Pawlikowski, B., Vogler, T. O., Gadek, K., and Olwin, B. B. (2017). Regulation of skeletal muscle stem cells by fibroblast growth factors. Dev. Dyn. 246, 359-367. doi: $10.1002 /$ dvdy.24495

Peserico, A., and Simone, C. (2011). Physical and functional hat/hdac interplay regulates protein acetylation balance. J. Biomed. Biotechnol. 2011:371832. doi: $10.1155 / 2011 / 371832$

Pigna, E., Renzini, A., Greco, E., Simonazzi, E., Fulle, S., Mancinelli, R., et al. (2018). HDAC4 preserves skeletal muscle structure following long-term denervation by mediating distinct cellular responses. Skelet. Muscle 8:6. doi: 10.1186/s13395018-0153-2

Puri, P. L., Iezzi, S., Stiegler, P., Chen, T. T., Schiltz, R. L., Muscat, G. E., et al. (2001). Class I histone deacetylases sequentially interact with $\mathrm{MyoD}$ and $\mathrm{pRb}$ during skeletal myogenesis. Mol. Cell 8, 885-897. doi: 10.1186/s13395-018-0153-2

Quinn, L. S., Anderson, B. G., Drivdahl, R. H., Alvarez, B., and Argilés, J. M. (2002). Overexpression of interleukin-15 induces skeletal muscle hypertrophy in vitro: implications for treatment of muscle wasting disorders. Exp. Cell Res. 280, 55-63. doi: 10.1016/S1097-2765(01)00373-2

Quinn, M. E., Goh, Q., Kurosaka, M., Gamage, D. G, Petrany, M. J, Prasad, V., et al. (2017). Myomerger induces fusion of non-fusogenic cells and is required for skeletal muscle development. Nat. Commun. 8:15665. doi: 10.1038/ ncomms 15665

Reisz-Porszasz, S., Bhasin, S., Artaza, J. N., Shen, R., Sinha-Hikim, I., Hogue, A., et al. (2003). Lower skeletal muscle mass in male transgenic mice with musclespecific overexpression of myostatin. Am. J. Physiol. Metab. 285, E876-E888. doi: 10.1152/ajpendo.00107.2003

Relaix, F., Montarras, D., Zaffran, S., Gayraud-Morel, B., Rocancourt, D., Tajbakhsh, S., et al. (2006). Pax3 and Pax7 have distinct and overlapping functions in adult muscle progenitor cells. J. Cell Biol. 172, 91-102.

Seale, P., Sabourin, L. A., Girgis-Gabardo, A., Mansouri, A., Gruss, P., and Rudnicki, M. A. (2000). Pax7 is required for the specification of myogenic satellite cells. Cell 102, 777-786. doi: 10.1083/jcb.200508044

Sun, Y., Ge, Y., Drnevich, J., Zhao, Y., Band, M., and Chen, J. (2010). Mammalian target of rapamycin regulates miRNA-1 and follistatin in skeletal myogenesis. J. Cell Biol. 189, 1157-1169. doi: 10.1083/jcb.200912093

Torrente, Y., Tremblay, J. P., Pisati, F., Belicchi, M., Rossi, B., Sironi, M., et al. (2001). Intraarterial injection of muscle-derived CD34(+)Sca-1(+) stem cells restores dystrophin in mdx mice. J. Cell Biol. 152, 335-348. doi: 10.1083/jcb. 200912093

Wang, Y. X., and Rudnicki, M. A. (2012). Satellite cells, the engines of muscle repair. Nat. Rev. Mol. Cell Biol. 13, 127-133. doi: 10.1038/nrm3265 
Williams, A. H., Valdez, G., Moresi, V., Qi, X., McAnally, J., Elliott, J. L., et al. (2009). MicroRNA-206 delays ALS progression and promotes regeneration of neuromuscular synapses in mice. Science 326, 1549-1554. doi: 10.1126/science. 1181046

Winbanks, C. E., Wang, B., Beyer, C., Koh, P., White, L., Kantharidis, P., et al. (2011). TGF-beta regulates miR-206 and miR-29 to control myogenic differentiation through regulation of HDAC4. J. Biol. Chem. 286, 13805-13814. doi: $10.1074 /$ jbc.M110.192625

Yin, H., Price, F., and Rudnicki, M. A. (2013). Satellite cells and the muscle stem cell niche. Physiol. Rev. 93, 23-67. doi: 10.1152/physrev. 00043.2011
Conflict of Interest Statement: The authors declare that the research was conducted in the absence of any commercial or financial relationships that could be construed as a potential conflict of interest.

Copyright (c) 2018 Renzini, Marroncelli, Noviello, Moresi and Adamo. This is an open-access article distributed under the terms of the Creative Commons Attribution License (CC BY). The use, distribution or reproduction in other forums is permitted, provided the original author(s) and the copyright owner(s) are credited and that the original publication in this journal is cited, in accordance with accepted academic practice. No use, distribution or reproduction is permitted which does not comply with these terms. 